\title{
ON LINEAR RELATIONS IN AN INDEFINITE INNER PRODUCT SPACE
}

\author{
PEKKA SORJONEN
}

\section{Introduction}

In 1961 R. Arens [2] initiated a study of linear relations, i.e. subspaces of $\mathfrak{H} \oplus \mathfrak{H}$, where $\mathfrak{H}$ is a Hilbert space. Since then there has been a growing interest in this subject; see for instance C. Bennewitz [4], E. A. Coddington [6-9], A. Dijksma and H. S. V. de Snoo [11], H. Langer and B. Textorius [18], A. Pleijel [19].

Some of these authors have used this theory mainly in studying differential equations which lead to linear relations in a Hilbert space.

On the other hand, there are some recent publications studying differential equations with an indefinite weight function which lead to symmetric operators in an indefinite inner product space; see F. V. Atkinson, W. N. Everitt and K. S. Ong [3], K. Daho and H. Langer [10] and H. Langer [17].

In this context a question arises: Can the theory of linear relations in a Hilbert space be extended to a corresponding theory in an indefinite inner product space? This paper tries to answer this question. In another publication we shall apply this theory to study cannonical differential equations with an indefinite weight function.

Chapter 1 summarizes the basic definitions and results of the theory of indefinite inner product spaces mainly because our terminology differs from that used by J. Bognár in [5], which is our main reference in indefinite inner product spaces.

Chapter 2 starts the study of linear relations in an indefinite inner product space: Section 2 contains a detailed investigation of reducing a linear relation to an operator; some of these results may be new also in the Hilbert space case. Section 3 represents a linear relation in a Krein space as a linear relation with a similar structure in a Hilbert space perturbed by an operator; these results extend the known facts of the operator case. In Section 4 we study the Cayley transformation. In Section 5 we generalize the notion of the operator matrix to linear relations.

Chapter 3 analyzes dissipative and maximal dissipative linear relations mostly along the lines of [11]. Some of these results seem to be new also in the operator case; compare with [15]. 
In Chapter 4 we give a detailed investigation of the basic properties of symmetric linear relations mostly in a Pontrjagin space. Eigenvalues and points of regular type are studied in Section 1. With the help of the relation matrix we characterize in Section 2 self-adjoint extensions of a symmetric linear relation in a Pontrjagin space. Section 3 introduces the deficiency spaces and the defects numbers.

\section{Indefinite inner product spaces}

1.1. Geometry. An (indefinite, non-degenerate) inner product space $\mathfrak{H}$ is a (complex) vector space with a non-degenerate hermitean sesquilinear form $[\cdot \mid \cdot]$. An element $f \in \mathfrak{H}$ (a subspace $\mathfrak{L} \subset \mathfrak{H}$ ) is said to be positive/non-negative/neutral/nonpositive/negative if $[f \mid f]>0 / \geqq 0 /=0 / \leqq 0 /<0$ (for all $f \in \mathfrak{L} \backslash\{0\}$ ).

Two vectors $f, g \in \mathfrak{H}$ (subspaces $\mathfrak{Q}, \mathfrak{M} \subset \mathfrak{S}$ ) are called orthogonal, written $f \perp g(\mathfrak{L} \perp \mathfrak{M})$ ), if $[f \mid g]=0$ (for all $f \in \mathfrak{L}, g \in \mathfrak{M}$ ). For a subspace $\mathfrak{L} \subset \mathfrak{H}$ we define $\mathfrak{L}^{\perp}:=\{f \in \mathfrak{H} \mid f \perp \mathfrak{L}\}$ and call it the orthogonal companion of $\mathfrak{L}$ in $\mathfrak{H}$. The set $\mathfrak{L}^{0}:=\mathfrak{L} \cap \mathfrak{L}^{\perp}$ is the isotropic part of $\mathfrak{L}$. If $\mathfrak{L}^{0} \neq\{0\}$, the subspace $\mathfrak{L}$ is called degenerate.

Following [2] we define the closure $\overline{\mathfrak{L}}$ of a subspace $\mathfrak{L}$ to be $\mathfrak{L}^{\perp \perp}$. The subspace $\mathfrak{Q}$ is called closed if $\overline{\mathfrak{Q}}=\mathfrak{Q}$ and dense if $\overline{\mathfrak{L}}=\mathfrak{H}$.

1.2. Operators. We use the following notations with a (linear) operator $T: \mathfrak{D}(T)$ is the domain, $\mathfrak{R}(T)$ the range and $\mathfrak{N}(T)$ the null space of $T$.

Let $T$ be an operator in an inner product space $\mathfrak{H}$. $T$ is dissipative if $\operatorname{Im}[T f \mid f] \geqq 0$ for all $f \in \mathfrak{D}(T) ; T$ is symmetric if $\operatorname{Im}[T f \mid f]=0$ or equivalently $[T f \mid g]=[f \mid T g]$ for all $f, g \in \mathfrak{D}(T)$. Let $\mathfrak{\Omega}$ be another inner product space and $T$ an operator from $\mathfrak{H}$ into $\mathfrak{S}$. $T$ is called contractive if $[T f \mid T f] \leqq[f \mid f]$ for all $f \in \mathfrak{D}(T)$, and isometric if $[T f \mid T f]=[f \mid f]$ or equivalently $[T f \mid T g]=[f \mid g]$ for all $f, g \in \mathfrak{D}(T)$. An isometric operator $T$ in $\mathfrak{H}$ is unitary if $\mathfrak{D}(T)=\mathfrak{R}(T)=\mathfrak{H}$. An orthogonal projector is a symmetric operator $T$ in $\mathfrak{H}$ with the properties $\mathfrak{D}(T)=\mathfrak{H}$ and $T^{2}=T$.

If $T$ is a densely defined operator from $\mathfrak{H}$ into $\mathfrak{R}$, then one can define the adjoint $T^{+}$of $T: \mathfrak{D}\left(T^{+}\right)$is the set of all those vectors $g \in \mathfrak{R}$ for which there exists a vector $h \in \mathfrak{H}$ such that $[T f \mid g]=[f \mid h]$ for all $f \in \mathfrak{D}(T)$, and then $T^{+} g:=h$. A densely defined operator $T$ in $\mathfrak{H}$ is called self-adjoint if $T^{+}=T$.

1.3. Fundamental decompositions. Let $\mathfrak{H}$ be an inner product space. It is said to be decomposable if it can be represented in the form

$$
\mathfrak{H}=\mathfrak{H}_{+}[\dot{+}] \mathfrak{H}_{-},
$$

where $\mathfrak{H}_{+} / \mathfrak{S}_{-}$is a positive/negative subspace. Here the symbol $[\dot{+}]$ denotes a direct and orthogonal sum. Every decomposition of this type is called a fundamental decomposition of $\mathfrak{H}$. The decomposition (1.1) induces so-called fundamental projectors $P_{+}$and $P_{-}: P_{ \pm} f:=f_{ \pm}$, where $f=f_{+}+f_{-} \in \mathfrak{H}$ with $f_{ \pm} \in \mathfrak{H}_{ \pm}$. The corresponding fundamental symmetry $J:=P_{+}-P_{-}$is then self-adjoint and unitary. 
With the help of a fundamental symmetry $J$ one can define $J$-inner product $(\cdot \mid \cdot)$ :

$$
(f \mid g):=[J f \mid g] \quad(f, g \in \mathfrak{H})
$$

which is positive definite, i.e. $\mathfrak{S}$ is a pre-Hilbert space with respect to the form $(\cdot \mid \cdot)$. For the corresponding norm, the so-called $J$-norm, $\|\cdot\|: f \mapsto(f \mid f)^{1 / 2}$ we have

$$
|[f \mid g]| \leqq\|f\|\|g\| \quad(f, g \in \mathfrak{H}) .
$$

When necessary, we use the prefix $J$ to denote a property which is defined using a $J$-inner product instead of an indefinite inner product. Thus we can speak about $J$-symmetric operators etc. Especially, $J$-adjoint is denoted by $*$ instead of + .

1.4. Krein spaces and Pontrjagin spaces. If a decomposable inner product space $\mathfrak{H}$ has a decomposition $(1.1)$ such that $\mathfrak{H}_{+}\left(\right.$resp. $\left.\mathfrak{H}_{-}\right)$is a Hilbert space with respect to the form $[\cdot \mid \cdot](-[\cdot \mid \cdot])$, it is called a Krein space. In this case $\mathfrak{H}$ is a Hilbert space with respect to every $J$-inner product and the $J$-norms are all equivalent. All topological notions in a Krein space are to be understood to refer to this $J$-norm topology. As the $J$-norm closure of a subspace $\mathfrak{L}$ is given by $\mathfrak{L}^{\perp \perp}$, our earlier terminology is consistent with the agreement just made. Note that a closed subspace $\mathfrak{L}$ is ortho-complemented, i.e. $\mathfrak{L}+\mathfrak{L}^{\perp}=\mathfrak{H}$, if and only if $\mathfrak{L}$ itself is a Krein space.

A Krein space $\mathfrak{H}$ with a fundamental decomposition (1.1) is called a Pontrjagin space (with $\varkappa$ negative squares) or a $\pi_{\varkappa}$-space if $\operatorname{dim} \mathfrak{H}_{-}=\varkappa$. In a Pontrjagin space a closed subspace is ortho-complemented if and only if it is non-degenerate.

1.5. Product spaces. Let $\mathfrak{H}$ and $\mathfrak{R}$ be inner product spaces. The product space $\mathfrak{H} \oplus \mathfrak{S}$ equipped with the usual linear structure and with the inner product

$$
[(f, g) \mid(h, k)]:=[f \mid h]+[g \mid k] \quad((f, g),(h, k) \in \mathfrak{H} \oplus \mathfrak{R})
$$

is also an inner product space.

Proposition 1.1. $1^{\circ}$ If $\mathfrak{H}$ and $\mathfrak{R}$ are decomposable with decompositions (1.1) and $\mathfrak{R}=\mathfrak{R}_{+}[\dot{+}] \mathfrak{H}_{-}$, then $\mathfrak{H} \oplus \mathfrak{K}$ is also decomposable and has a fundamental decomposition $^{1}$

$$
\mathfrak{H} \oplus \mathfrak{R}=\mathfrak{H}_{+} \oplus \mathfrak{R}_{+}[\dot{+}] \mathfrak{H}_{-} \oplus \mathfrak{R}_{-} .
$$

$2^{\circ}$ If $\mathfrak{H}$ and $\mathfrak{H}$ are Krein spaces, then $\mathfrak{H} \oplus \mathfrak{K}$ is also a Krein space.

$3^{\circ}$ If $\mathfrak{H}$ is a $\pi_{\varkappa}$-space and $\mathfrak{R}$ is a $\pi_{\varkappa^{\prime}}$-space, then $\mathfrak{H} \oplus \mathfrak{R}$ is a Pontrjagin space with $\varkappa+\varkappa^{\prime}$ negative squares.

The proof is clear from the definitions.

1 The symbol + means the algebraic sum in the product space. 


\section{Linear relations}

2.1. Preliminaries. Throughout this chapter $(\mathfrak{b}, \mathfrak{H}$ and $\mathfrak{S}$ are inner product spaces.

A linear relation from $\mathfrak{H}$ into $\mathfrak{S}$ is a subspace $T$ of the product space $\mathfrak{H} \oplus \mathfrak{\Omega}$; if $\mathfrak{i}=\mathfrak{H}, T$ is said to be a linear relation in $\mathfrak{H}$. A linear relation is closed if it is a closed subspace. We recall the following definitions and notations for linear relations $T$ and $S$ from $\mathfrak{H}$ into $\mathfrak{S}$ and $R$ from $\mathfrak{b}$ into $\mathfrak{H}$ :

$$
\begin{aligned}
\mathfrak{D}(T) & :=\{f \in \mathfrak{H} \mid(f, g) \in T \text { for some } g \in \mathfrak{N}\}, \\
\mathfrak{R}(T) & :=\{g \in \mathfrak{N} \mid(f, g) \in T \text { for some } f \in \mathfrak{S}\}, \\
\mathfrak{N}(T) & :=\{f \in \mathfrak{H} \mid(f, 0) \in T\}, \\
T(f) & :=\{g \in \mathfrak{S} \mid(f, g) \in T\} \quad(f \in \mathfrak{D}(T)), \\
T^{-1} & :=\{(g, f) \in \mathfrak{i} \oplus \mathfrak{H} \mid(f, g) \in T\}, \\
z T & :=\{(f, z g) \in \mathfrak{H} \oplus \mathfrak{N} \mid(f, g) \in T\} \quad(z \in C:=\text { complex numbers }), \\
S+T & :=\{f, g+k) \in \mathfrak{H} \oplus \mathfrak{N} \mid(f, g) \in S,(f, k) \in T\}, \\
S R & :=S \circ R:=\{(f, k) \in \mathfrak{G} \oplus \mathfrak{N} \mid(f, g) \in R,(g, k) \in S \text { for some } g \in \mathfrak{S}\}, \\
T^{+} & :=\{(k, h) \in \mathfrak{N} \oplus \mathfrak{H} \mid[f \mid h]=[g \mid k] \text { for all }(f, g) \in T\} .
\end{aligned}
$$

The only new definition here compared to the Hilbert space case (see [6] and [11]) is the adjoint, which has been formed with respect to the (indefinite) inner product. But because this inner product gives a duality, our definition of the adjoint is a particular case from [2].

The basic algebraic properties of linear relations are given in [2]; we list here only the following facts about the adjoint:

$$
\begin{gathered}
T^{+} \quad \text { is closed, } \quad \bar{T}=T^{++}, \\
S \subset T \text { implies } \quad T^{+} \subset S^{+}, \\
(z T)^{+}=\bar{z} T^{+} \quad(z \in C), \\
\left(T^{-1}\right)^{+}=\left(T^{+}\right)^{-1}, \\
\mathfrak{N}\left(T^{+}\right)=\mathfrak{R}(T)^{\perp}, \quad T^{+}(0)=\mathfrak{D}(T)^{\perp} .
\end{gathered}
$$

If we identify an operator $T$ from $\mathfrak{H}$ into $\mathfrak{K}$ with its graph in $\mathfrak{G} \oplus \mathfrak{R}$, it is easily seen that a linear relation $T$ is an operator iff (if and only if) $T(0)=\{0\}$. Note that two relations $S$ and $T$ with $\mathfrak{D}(S)=\mathfrak{D}(T)$ and $S(0)=T(0)$ are equal iff $S \subset T$.

As in the case of linear operators the following linear relations $T$ in $\mathfrak{H}$ are of interest: 
(i) dissipative linear relations, i.e. $\operatorname{Im}[g \mid f] \geqq 0$ for all $(f, g) \in T$;

(ii) symmetric linear relations, i.e. $\operatorname{Im}[g \mid f]=0$ for all $(f, g) \in T$ or equivalently $T \subset T^{+}$;

(iii) self-adjoint linear relations, i.e. $T=T^{+}$;

(iv) contractive linear relations, i.e. $[g \mid g] \leqq[f \mid f]$ for all $(f, g) \in T$;

(v) isometric linear relations, i.e. $[g \mid g]=[f \mid f]$ for all $(f, g) \in T$;

(vi) unitary linear relations, i.e. $T$ is isometric and $\mathfrak{D}(T)=\mathfrak{R}(T)=\mathfrak{H}$.

One can define in the usual way a maximal dissipative linear relation etc. Of course the definitions (iv) and (v) can be extended to linear relations from $\mathfrak{S}$ into $\mathfrak{\Omega}$.

If the space $\mathfrak{H}$ is a Hilbert space, the last three linear relations are not interesting as relations because they are all operators, but this is not the case in a general inner product space: Arens [2] has proved that a linear relation $T$ is isometric iff $T^{-1} \subset T^{+}$, which implies $T(0) \subset \mathfrak{R}(T)^{0}$ for an isometric relation $T$. Hence in our case, where the inner product spaces are supposed to be non-degenerate, all unitary relations are operators. On the other hand, an isometric relation is not necessarily an operator. A simple counter-example is given by the relation $T:=\{0\} \oplus \mathfrak{Q}$, where $\mathfrak{L} \neq\{0\}$ is a neutral subspace in a Pontrjagin space.

We shall need the following result, which is known in the Hilbert space case and also for densely defined operators in a Krein space; see [5].

Proposition 2.1. Let $T$ be a closed linear relation from a Krein space $\mathfrak{H}$ into a Krein space $\mathfrak{R}$. Then $\mathfrak{R}(T)$ is closed iff $\mathfrak{R}\left(T^{+}\right)$is closed.

Proof. It is enough to prove that $\mathfrak{R}\left(T^{+}\right)$is closed if $\mathfrak{R}(T)$ is closed, because the converse follows from $T=T^{++}$. On the other hand, $\mathfrak{R}(T)$ is closed (in $\mathfrak{H}$ ) iff $\mathfrak{H} \oplus \mathfrak{R}(T)=T+\mathfrak{H} \oplus\{0\}$ is closed (in $\mathfrak{H} \oplus \mathfrak{H}$ ), and $\mathfrak{R}\left(T^{+}\right)$is closed iff $T^{\perp}+(\mathfrak{H} \oplus\{0\})^{\perp}$ is closed. So it is more than enough to prove the identity

$$
T^{\perp}+S^{\perp}=(T \cap S)^{\perp}
$$

for linear relations $T$ and $S$, for which the sum $T+S$ is closed. If one uses the duality given by the inner product and the Hahn-Banach theorem, this result follows by a light modification of the proof of the corresponding fact in a Banach space; see [14], Lemma IV-4.9.

Let $T$ be a linear relation in $\mathfrak{H}$. Every $z \in C$ for which $(f, z f) \in T$ with some $f \neq 0$ is called an eigenvalue of $T$; the corresponding vectors $f$ are eigenvectors belonging to the eigenvalue $z$. The set of all eigenvalues of $T$ is denoted by $\sigma_{p}(T)$. If for some $z \in C$ the relation $(T-z I)^{-1}$ is an everywhere defined operator, then $z$ belongs to the resolvent set $\varrho(T)$ of $T$. (Here and in the following $I$ denotes the identity operator or the corresponding linear relation.) The spectrum $\sigma(T)$ of $T$ is the complement of $\varrho(T)$ in $C$.

To be able to put some later results in a concise form we introduce the notion of a point of regular type. For this let $T$ be a linear relation in a Krein space $\mathfrak{H}$. 
A complex number $z$ is called a point of regular type of the relation $T$ if there exists a constant $c:=c(z)>0$ such that $\|g-z f\| \geqq c\|f\|$ for all $(f, g) \in T$. The set of all points of regular type of $T$ is denoted by $r(T)$.

Theorem 2.2. Let $T$ be a linear relation in a Krein space $\mathfrak{H}$. Then $c(z)^{-1}$;

(i) $(T-z I)^{-1}$ is a continuous operator iff $z \in r(T)$; in this case $\left\|(T-z I)^{-1}\right\| \leqq$

(ii) if $\mathfrak{R}(T-z I)$ is closed for one $z \in r(T)$, then $T$ is closed;

(iii) $r(T)$ is open.

If in addition $T$ is closed, then for all $z \in r(T)$

(iv) $\mathfrak{R}(T-z I)$ is closed;

(v) $\mathfrak{R}(T-z I)=\mathfrak{N}\left(T^{+}-z I\right)^{\perp}$;

(vi) $\mathfrak{R}\left(T^{+}-\bar{z} I\right)=\mathfrak{H}$.

Proof. (i) and (iii) can be proved in the same way as in the operator case; see e.g. [1], Nr. 100. With a light modification of the proof of Theorem 3.1 in [11] we get the other claims.

2.2. Reduction to an operator. A useful method of studying linear relations is to reduce them to operators. To do this we need first some facts about the (purely) multi-valued part $T_{\infty}:=\{(0, g) \in T\}$ of a linear relation $T$.

Proposition 2.3. If $T$ is a linear relation from $\mathfrak{H}$ into $\mathfrak{\Omega}$, then

(i) $T_{\infty}=\{0\} \oplus T(0)$;

(ii) $\mathfrak{D}\left(T_{\infty}\right)=\{0\}, \mathfrak{R}\left(T_{\infty}\right)=T(0)$;

(iii) $\left(T_{\infty}\right)^{\perp}=\mathfrak{H} \oplus T(0)^{\perp},\left(T_{\infty}\right)^{+}=T(0)^{\perp} \oplus \mathfrak{H}$;

(iv) $\bar{T}(0)=\mathfrak{D}\left(T^{+}\right)^{\perp}, \overline{\mathfrak{D}(\bar{T})}=T^{+}(0)^{\perp}$;

(v) $T_{\infty}$ is closed/ortho-complemented iff $T(0)$ is closed/ortho-complemented;

(vi) $T_{\infty}$ is non-degenerate iff $T(0)$ is non-degenerate iff $T \cap\left(T_{\infty}\right)^{\perp}$ is an operator. If in addition $\mathfrak{R}=\mathfrak{H}$, then

(vii) $T_{\infty}$ is symmetric;

(viii) $(T-z I)_{\infty}=T_{\infty}, z \in C$.

Proof. (i) - (iv) and (vii)-(viii) are obvious from the corresponding Hilbert space results; see [6] and [11]. (v) and (vi) follow from (i), (iii) and from the easily verifiable identities

$$
\begin{gathered}
\left(T_{\infty}\right)^{\perp \perp}=\{0\} \oplus T(0)^{\perp \perp}, \\
\left(T_{\infty}\right)^{0}=\{0\} \oplus T(0)^{0}=\{0\} \oplus\left(T \cap\left(T_{\infty}\right)^{\perp}\right)(0) .
\end{gathered}
$$

Theorem 2.4. Let $T$ be a linear relation from $\mathfrak{H}$ into $\mathfrak{\Omega}$ with ortho-complemented $T_{\infty}$. Then $T_{s}:=T \cap\left(T_{\infty}\right)^{\perp}$ is an operator with $\mathfrak{D}\left(T_{s}\right)=\mathfrak{D}(T)$ and $\mathfrak{R}\left(T_{s}\right) \subset T(0)^{\perp}$, and

$$
T=T_{s}[\dot{+}] T_{\infty} .
$$




\section{Furthermore,}

(i) $T_{s}$ is closed if (iff, in case $\mathfrak{S}$ and $\mathfrak{S}$ are Krein spaces) $T$ is closed;

(ii) $T_{s}$ is ortho-complemented iff $T$ is ortho-complemented;

(iii) $\mathfrak{R}(T)=\mathfrak{R}\left(T_{s}\right)[+] T(0)$.

Proof. As $T_{\infty}$ is ortho-complemented, it is non-degenerate, and hence $T_{s}$ is an operator according to Proposition 2.3. The decomposition (2.1) is a consequence of the decomposition $\mathfrak{H} \oplus \mathfrak{K}=T_{\infty}[\dot{+}]\left(T_{\infty}\right)^{\perp}$. The relations concerning the domain and the range as well as (i)-(iii) follows from (2.1) and Proposition 2.3. The "iff" in (i) follows from [16], Lemma 5.1.

As to the uniqueness of the decomposition given above we have

Proposition 2.5. Let $T$ be a linear relation from $\mathfrak{H}$ into $\mathfrak{R}$ and $S$ an operator from $\mathfrak{S}$ into $\mathfrak{K}$ such that $T=S[+] T_{\infty}$. Then $S=T_{s}$ iff $T_{\infty}$ is non-degenerate.

Proof. $1^{\circ}$ If $S=T_{s}$, then every $(0, k) \in\left(T_{\infty}\right)^{0}$ belongs to $T \cap\left(T_{\infty}\right)^{\perp}=T_{s}=S$ so that $k \in S(0)=\{0\}$; hence $T_{\infty}$ is non-degenerate.

$2^{\circ}$ Let $T_{\infty}$ be non-degenerate. As $S \subset T \cap\left(T_{\infty}\right)^{\perp}=T_{s}$, it is enough to prove that every $(f, g) \in T_{s}(\subset T)$ belongs to $S$. We have $(f, g)=(f, S f)+(0, k)$ with $k \in T(0)$; hence

$$
\begin{aligned}
{[k \mid l] } & =[g-S f \mid l]=[(f, g)-(f, S f) \mid(0, l)] \\
& =[(f, g) \mid(0, l)]=0
\end{aligned}
$$

for all $l \in T(0)$, because $S \perp T_{\infty}$ and $T_{s} \perp T_{\infty}$. This means that $k \in T(0) \cap T(0)^{\perp}=\{0\}$ (by Proposition 2.3), but then $(f, g)=(f, S f) \in S$.

The preceeding theorem gives a reduction of a linear relation $T$ in $\mathfrak{H}$ to the operator $T_{s}$ in $\mathfrak{H}$, but usually $\mathfrak{H}$ is too "large". The following result tells us when $T_{s}$ is an operator in $T(0)^{\perp}$.

Proposition 2.6. Let $T$ be a linear relation in $\mathfrak{H}$ with ortho-complemented $T_{\infty}$. If $\mathfrak{R}\left(T_{s}-z I\right) \subset T(0)^{\perp}$ for one $z \in C \backslash\{0\}$, then $\mathfrak{D}\left(T_{s}\right) \subset T(0)^{\perp}$. Conversely, if $\mathfrak{D}\left(T_{s}\right) \subset T(0)^{\perp}$, then $\mathfrak{R}\left(T_{s}-z I\right) \subset T(0)^{\perp}$ for all $z \in C$.

Proof. For all $f \in \mathfrak{D}\left(T_{s}\right), g \in T(0)$ and $z \in C \backslash\{0\}$ we have

$$
[f \mid g]=-z^{-1}\left[\left(T_{s}-z I\right) f \mid g\right]
$$

from which the result follows.

Next we shall study how much information is retained by this reduction to an operator.

Theorem 2.7. Let $T$ be a linear relation in $\mathfrak{H}$ such that $T_{\infty}$ is ortho-complemented and $\mathfrak{D}(T) \subset T(0)^{\perp}$. Regard $T_{s}$ as an operator in $T(0)^{\perp}$ and let $z \in C$. Then 
(i) $T-z I=\left(T_{s}-z I\right)[\dot{+}] T_{\infty}$;

(ii) $\mathfrak{R}(T-z I)=\mathfrak{R}\left(T_{s}-z I\right)[+] T(0)$;

(iii) $\mathfrak{R}\left(T_{s}-z I\right)$ is closed if (iff, in a Krein space) $\mathfrak{R}(T-z I)$ is closed;

(iv) $\mathfrak{R}\left(T_{s}-z I\right)$ is ortho-complemented iff $\mathfrak{R}(T-z I)$ is ortho-complemented;

(v) $\mathfrak{N}(T-z I)=\mathfrak{N}\left(T_{s}-z I\right)$;

(vi) $\sigma_{p}(T)=\sigma_{p}\left(T_{s}\right)$ and for $z \notin \sigma_{p}(T)$ the relation $\left(T_{s}-z I\right)^{-1}$ is an operator, and

$$
(T-z I)^{-1}=\left(T_{s}-z I\right)^{-1}[\dot{+}] O_{T(0)},
$$

where $O_{T(0)}$ denotes the zero operator in $T(0)$.

In addition let $\mathfrak{H}$ be a Krein space. Then of $\mathfrak{S}$;

(vii) $r(T)=r\left(T_{s}\right)$ in case $T(0) \subset \mathfrak{H}_{+}$for a fundamental decomposition (1.1)

(viii) $\sigma(T)=\sigma\left(T_{s}\right)$ in case $T$ is closed.

The proof is mostly a direct calculation.

The following results show that we can use this reduction to investigate all the interesting linear relations.

Lemma 2.8. Let $T$ be a dissipative linear relation in $\mathfrak{H}$. Then $\mathfrak{D}(T) \subset T(0)^{\perp}$.

Proof. Let $f \in \mathfrak{D}(T)$ and $k \in T(0)$ be arbitrary and let $g \in T(f)$. Then $z k+g \in T(0)+g=T(f)$ for all $z \in C$. Hence $(f, z k+g) \in T$ and so

$$
0 \leqq \operatorname{Im}(z[k \mid f])+\operatorname{Im}[g \mid f] .
$$

Thus $-\operatorname{Im}(z[k \mid f])$ is bounded above with the non-negative constant $\operatorname{Im}[g \mid f]$ for all $z \in C$, but this is possible only if $[k \mid f]=0$, i.e. $T(0) \perp \mathfrak{D}(T)$.

Theorem 2.9. Let $T$ be a linear relation in $\mathfrak{H}$ such that $T_{\infty}$ is ortho-complemented. Then

(i) $T$ is a dissipative relation iff $T_{s}$ is a dissipative operator in $T(0)^{\perp}$;

(ii) $T$ is a symmetric relation iff $T_{s}$ is a symmetric operator in $T(0)^{\perp}$;

(iii) $T$ is a self-adjoint relation iff $T_{s}$ is a densely defined self-adjoint operator in $T(0)^{\perp}$;

(iv) $T$ is a contractive relation iff $T(0)$ is non-positive and $T_{s}$ is a contractive operator;

(v) $T$ is an isometric relation iff $T$ is an isometric operator.

Proof. (i) If $T_{s}$ is a dissipative operator in $T(0)^{\perp}$, then

$$
\operatorname{Im}[g \mid f]=\operatorname{Im}\left[T_{s} f+k \mid f\right]=\operatorname{Im}\left[T_{s} f \mid f\right] \geqq 0
$$

for all $(f, g)=\left(f, T_{s} f\right)+(0, k) \in T=T_{s}[\dot{+}] T_{\infty}$. Conversely, if $T$ is a dissipative relation, it is clear that $T_{s}$ is a dissipative operator in $T(0)^{\perp}$ (see Lemma 2.8).

(ii) Suppose that $T_{s}$ is symmetric in $T(0)^{\perp}$. Then

$$
T_{s} \subset\left(T_{s}\right)^{+}, \quad T_{\infty} \subset\left(T_{\infty}\right)^{+}, \quad T_{s} \subset T(0)^{\perp} \oplus \mathfrak{H}=\left(T_{\infty}\right)^{+}
$$


(see Proposition 2.3). These together imply that $T=T_{s}[\dot{+}] T_{\infty}$ is symmetric. The other half follows from (i) and the fact $T_{s} \subset T$.

(iii) If $T$ is self-adjoint, then $T_{s}$ is symmetric by (ii) and $\mathfrak{D}\left(T_{s}\right)=\mathfrak{D}(T)=\mathfrak{D}(\bar{T})$ is dense in $T(0)^{\perp}$ by Proposition 2.3. Hence the adjoint of $T_{s}$ exists as an operator in the space $T(0)^{\perp}$ and $T_{s} \subset\left(T_{s}\right)^{+}$. For all $h \in \mathfrak{D}\left(\left(T_{s}\right)^{+}\right)$and $\left(T_{s} f+k, g\right) \in T=$ $T_{s}[\dot{\dagger}] T_{\infty}$ one obtains

$$
\left[T_{s} f+k \mid h\right]=\left[T_{s} f \mid h\right]=\left[f \mid\left(T_{s}\right)^{+} h\right],
$$

and so $\left(h,\left(T_{s}\right)^{+} h\right) \in T^{+}=T$. This implies $h \in \mathfrak{D}(T)=\mathfrak{D}\left(T_{s}\right)$, i.e. $\left(T_{s}\right)^{+}=T_{s}$.

Conversely, if $T_{s}$ is a self-adjoint operator in $T(0)^{\perp}$, then $T \subset T^{+}$by (ii). Furthermore, we have

$$
\left[T_{s} f+k \mid h\right]=[f \mid l]
$$

for all $(h, l) \in T^{+}$and $\left(f, T_{s} f+k\right) \in T_{s}[\dot{+}] T_{\infty}$. Especially for $k=0$,

$$
\left[T_{s} f \mid h\right]=\left[f \mid l_{2}\right]
$$

where $l=l_{1}+l_{2} \in T(0)[\dot{+}] T(0)^{\perp}$; hence $h \in \mathfrak{D}\left(\left(T_{s}\right)^{+}\right)$and $\left(T_{s}\right)^{+} h=T_{s} h=l_{2}$. This means that $(h, l)=\left(h, T_{s} h+l_{1}\right)$ is in $T_{s}[\dot{+}] T_{\infty}=T$.

(iv) If $T$ is a contractive relation, then

$$
\left[T_{s} f+k \mid T_{s} f+k\right] \leqq[f \mid f]
$$

for all $f \in \mathfrak{D}(T)=\mathfrak{D}\left(T_{s}\right), k \in T(0)$. By choosing $k=0$ or $f=0$ we get the result. The converse follows similarly.

(v) Let $T$ be an isometric relation. Then we can replace in the proof of (iv) the inequality with the equality. This shows that $T_{s}$ is isometric and $T(0)$ is neutral. But $T_{\infty}$ is non-degenerate and hence $T(0)$ must be $\{0\}$ by Proposition 2.3.

Note that as $T(0)$, and with it also $T(0)^{\perp}$, is ortho-complemented, $T(0)^{\perp}$ is a Krein space/a Pontrjagin space if $\mathfrak{H}$ is a Krein space/a Pontrjagin space.

2.3. Reduction to a relation in a Hilbert space. In the previous section we reduced a linear relation $T$ in $\mathfrak{G}$ to an operator. Here we are going to show another reduction, which is more usable in the Krein space case.

The o rem 2.10. Let T be a linear relation in a Krein space $\mathfrak{Y}$ such that $\mathfrak{D}(T) \supset \mathfrak{H}$ for a fundamental decomposition (1.1) of $\mathfrak{h}$. Let $J$ be the corresponding fundamental symmetry. Define

$$
S:=T J=\{(f, g) \mid(J f, g) \in T\} .
$$

Then $S$ is a linear relation in $\mathfrak{H}$ with $\mathfrak{D}(S)=\mathfrak{D}(T), S(0)=T(0)$ and

$$
T=S+2 T P_{-},
$$

where $P_{-}$is the fundamental projector belonging to (1.1). Furthermore, 
(i) $S$ is closed iff $T$ is closed;

(ii) $T$ is dissipative/maximal dissipative/symmetric/maximal symmetric/self-adjoint iff $S$ is $J$-dissipative/maximal $J$-dissipative/J-symmetric/maximal $J$-symmetric/J-selfadjoint.

Proof. Since $\mathfrak{D}(T) \supset \mathfrak{G}$ - and $J^{2}=I$ it follows that $\mathfrak{D}(S)=\mathfrak{D}(T)$ and $S(0)=T(0)$.

Let us prove (2.3). Every $(f, g) \in S+2 T P_{-}$is of the form $(h, k+l)$, where $(J h, k),\left(2 P_{-} h, l\right) \in T$; then

$$
(f, g)=(h, k+l)=(J h, k)+\left(2 P_{-} h, l\right) \in T .
$$

Conversely, if $(f, g) \in T$, then $2 P_{-} f, J f \in \mathcal{D}(T)$. Hence $\left(2 P_{-} f, l\right) \in T$ or $(f, l) \in$

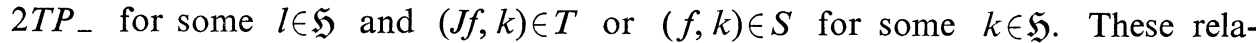
tions imply $(f, k+l)=(J f, k)+\left(2 P_{-} f, l\right) \in T$ and further $(0, g-k-l)=(f, g)-$ $(f, k+l) \in T$. So $(J f, g-l)=(J f, k)+(0, g-k-l)$ is in $T$ or $(f, g-l)$ is in $S$. Putting these facts together we see that $(f, g)=(f,(g-l)+l)$ is in $S+2 T P_{-}$.

(i) follows from the facts that $J$ is continuous and $T=S J$. (ii) is a direct calculation, which uses relation (1.2) and the basic properties of the fundamental symmetry $J$.

In case $S_{\infty}\left(=T_{\infty}\right)$ is $J$-ortho-complemented, i.e. closed, we can go a step further using Theorem 2.4 and reduce $T$ to the operator $S_{s}$ :

$$
T=\left(S_{s}(\dot{+}) S_{\infty}\right)+2 T P_{-} ;
$$

here $(+)$ means a sum which is orthogonal with respect to a $J$-inner product. We can put this decomposition in a more usable form:

Theorem 2.11. Let $T$ be a linear relation in a Krein space $\mathfrak{H}$ such that $T_{\infty}$ is closed and $\mathfrak{D}(T) \supset H_{-}$for a fundamental decomposition (1.1) of $\mathfrak{H}$ with the fundamental symmetry $J:=P_{+}-P_{-}$. Define $S:=T J, S_{s}:=S \cap\left(S_{\infty}\right)^{(\perp)}$ and $A:=-2 S_{s} P_{-}$. Then

$$
T=S+A, \quad T_{s}=S_{s}+A .
$$

Furthermore, parts (i) and (ii) of Theorem 2.10 remain true.

Proof. We verify the first identity in $\left(2.3^{\prime}\right)$; the second follows similarly. All other claims are obvious in the light of Theorem 2.10. Let $(f, g) \in T$. Then $(J f, g) \in S=$ $S_{s}(\dot{+}) S_{\infty}$ and so $(J f, g)=\left(h, S_{s} h\right)+(0, k)$ with $k \in S(0)$. Because $h=J f$ and $J-I=-2 P_{-}$, we get

$$
g=S_{s}((J-I)+I) f+k=A f+S_{s} f+k .
$$

Hence $(f, g)=\left(f,\left(S_{s} f+k\right)+A f\right)$ with $\left(f, S_{s} f+k\right) \in S_{s}(\dot{+}) S_{\infty}=S$ and so $(f, g) \in S+A$. this case

Conversely, let $(f, g) \in S+A$; then $(f, g)=\left(h, S_{s} h+k+A h\right)$ with $k \in S(0)$. In

$$
g=S_{s} f-2 S_{s} P_{-} f+k=S_{s} J f+k,
$$


which implies that the vector $(J f, g)=\left(J f, S_{s} J f\right)+(0, k)$ is in $S_{s}(\dot{+}) S_{\infty}=S$, i.e. $(f, g) \in T$.

Note that this theorem gives us (at least theoretically) the possibility to use the perturbation theory in studying a linear relation $T$ in a Krein space: $T$ can be regarded as the corresponding Hilbert space relation $S$ perturbed by the operator $A$.

2.4. The Cayley transformation. Following [11] we define for $z \in \boldsymbol{C}$ the Cayley transform $C_{z}(T)$ of a linear relation $T$ in $\mathfrak{H}$ by

$$
C_{z}(T):=\{(g-z f, g-\bar{z} f) \mid(f, g) \in T\}
$$

and the inverse transform $F_{z}(T)$ of $T$ by

$$
F_{z}(T):=\{(g-f, z g-\bar{z} f) \mid(f, g) \in T\}
$$

Then $C_{z}(T)$ and $F_{z}(T)$ are linear relations in $\mathfrak{H}$ with $\mathfrak{D}\left(C_{z}(T)\right)=\mathfrak{R}(T-z I)$, $\mathfrak{D}\left(F_{z}(T)\right)=\mathfrak{R}(T-I)$ and $\mathfrak{R}\left(C_{z}(T)\right)=\mathfrak{R}(T-\bar{z} I), \mathfrak{R}\left(F_{z}(T)\right)=\mathfrak{R}(z T-\bar{z} I)$. For future use we quote the following result from [11]:

Lemma 2.12. Let $T$ and $S$ be linear relations in $\mathfrak{S}$ and $z \in \boldsymbol{C} \backslash \boldsymbol{R}$. Then

(i) $T=C_{z}\left(F_{z}(T)\right)=F_{z}\left(C_{z}(T)\right)$;

(ii) $T \subset S$ iff $C_{z}(T) \subset C_{z}(S)$ iff $F_{z}(T) \subset F_{z}(S)$;

(iii) $C_{-z}(T)=C_{z}(-T), F_{-z}(T)=-F_{z}(T)$;

(iv) $C_{\bar{z}}(T)=C_{z}(T)^{-1}, F_{\bar{z}}(T)=F_{z}\left(T^{-1}\right)$;

(v) $C_{z}\left(T^{+}\right)=C_{\bar{z}}(T)^{+}, F_{z}\left(T^{+}\right)=F_{\bar{z}}(T)^{+}$;

(vi) $C_{z}(T+S)=C_{z}(T)+C_{z}(S), F_{z}(T+S)=F_{z}(T)+F_{z}(S)$ and the sums are direct iff the sum $T+S$ is direct;

(vii) $C_{z}(T)(0)=\mathfrak{N}(T-z I), F_{z}(T)(0)=\mathfrak{N}(T-I)$;

(viii) $\mathfrak{D}(T)=\mathfrak{R}\left(C_{z}(T)-I\right)=\mathfrak{R}\left(F_{z}(T)-z I\right)$;

(ix) $\mathfrak{R}(T)=\mathfrak{R}\left(C_{z}(T)-\bar{z} z^{-1} I\right)=\mathfrak{R}\left(F_{z}(T)-\bar{z} I\right)$;

(x) $T(0)=\mathfrak{N}\left(C_{z}(T)-I\right)=\mathfrak{N}\left(F_{z}(T)-z I\right)$;

(xi) in case $\mathfrak{H}$ is a Krein space, $T$ is closed iff $C_{z}(T)$ is closed iff $F_{z}(T)$ is closed.

Let $z$ be a non-real complex number. We define a mapping $c_{z}$ in the extended complex plane $\overline{\boldsymbol{C}}:=\boldsymbol{C} \cup\{\infty\}$ as follows:

$$
c_{z}(w):=\left\{\begin{array}{lll}
(w-\bar{z})(w-z)^{-1}, & \text { if } \quad w \in C \backslash\{z\} \\
\infty, & \text { if } \quad w=z, \\
1, & \text { if } \quad w=\infty
\end{array}\right.
$$

We agree to say that $\infty$ is an eigenvalue of a linear relation $T$ if $0 \in \sigma_{p}\left(T^{-1}\right)$, i.e. $T$ is not an operator. Then the spectral mapping theorem is true (compare with [2] and [18]): 
Proposition 2.13. Let $T$ be a linear relation in $\mathfrak{H}$ and $z \in \boldsymbol{C} \backslash \boldsymbol{R}$. Then

$$
c_{z}(\sigma(T))=\sigma\left(C_{z}(T)\right) .
$$

Moreover, eigenvalues correspond to eigenvalues.

The proof is clear if one uses Lemma 2.12 and the relation

$$
\mathfrak{R}\left(C_{z}(T)-c_{z}(w) I\right)=\mathfrak{R}(T-z I), \quad w \in C .
$$

We denote by $\boldsymbol{C}_{+}\left(\boldsymbol{C}_{-}\right)$the open upper (lower) half-plane of the complex plane $C$. As to the Hilbert space case of the following two results, see [11].

Theorem 2.14. Let $T$ be a linear relation in $\mathfrak{H}$.

(i) If $T$ is dissipative, then $C_{z}(T)$ is contractive for all $z \in C_{-}$and an operator for all $z \in \boldsymbol{C} \backslash \sigma_{p}(T)$.

(ii) If $T$ is symmetric, then $C_{z}(T)$ is isometric for all $z \in C$ and an operator for all $z \in \boldsymbol{C} \backslash \sigma_{p}(T)$.

(iii) If $\mathfrak{H}$ is a Krein space and $T$ is self-adjoint, then $C_{z}(T)$ is a unitary operator for all $z \in \varrho(T)$.

Proof. The identity

$$
[g-z f \mid g-z f]-[g-\bar{z} f \mid g-\bar{z} f]=-4(\operatorname{Im} z)[g \mid f]
$$

for all $(f, g) \in T$ and Lemma 2.12 imply (i) and (ii).

Because $\mathfrak{R}(T-z I)=\mathfrak{H}$ and $\mathfrak{N}(T-z I)=\{0\}$ in (iii), we have, by Proposition 2.1, $\mathfrak{D}\left(C_{z}(T)\right)=\mathfrak{R}(T-z I)=\mathfrak{H}$ and

$$
\begin{aligned}
\mathfrak{R}\left(C_{z}(T)\right) & =\mathfrak{R}(T-\bar{z} I)=\mathfrak{R}\left((T-z I)^{+}\right)=\mathfrak{R}\left((T-z I)^{+}\right)^{\perp \perp} \\
& =\mathfrak{N}(T-z I)^{\perp}=\mathfrak{H} ;
\end{aligned}
$$

hence $C_{z}(T)$ is unitary by (ii).

The most remarkable fact here is that the Cayley transform is not always an operator as in the Hilbert space case. Theorem 2.14 has the following converse.

Theorem 2.15. Let $T$ be a linear relation in $\mathfrak{H}$.

(i) If $T$ is contractive, then $F_{z}(T)$ is dissipative for all $z \in C_{-}$.

(ii) If $T$ is isometric, then $F_{z}(T)$ is symmetric for all $z \in C$.

(iii) If $T$ is unitary, then $F_{z}(T)$ is self-adjoint for all $z \in \boldsymbol{C} \backslash \boldsymbol{R}$.

Proof. The identity

$$
\operatorname{Im}[z g-\bar{z} f \mid g-f]=(\operatorname{Im} z)([g \mid g]-[f \mid f])
$$

for all $(f, g) \in T$ implies (i) and (ii). (iii) follows from (ii) and from the facts

$$
\mathfrak{R}\left(F_{z}(T)-z I\right)=\mathfrak{D}(T)=\mathfrak{H}=\mathfrak{R}(T)=\mathfrak{R}\left(F_{z}(T)-\bar{z} I\right)
$$

for all $z \in \boldsymbol{C} \backslash \boldsymbol{R}$; see Lemma 2.12. 
2.5. The relation matrix. In this section we suppose that $\mathfrak{H}$ is a Krein space. Let $T$ be a linear relation in $\mathfrak{H}$. Extending the notion of the operator matrix we say that $T$ is represented by the relation matrix

$$
\left[\begin{array}{ll}
T_{++} & T_{-+} \\
T_{+-} & T_{--}
\end{array}\right]
$$

with respect to the fundamental decomposition (1.1) of $\mathfrak{H}$ if $T_{ \pm \pm}$is a relation from $\mathfrak{H}_{ \pm}$into $\mathfrak{H}_{ \pm}$and

$$
T=\left(T_{++}+T_{-+}\right)+\left(T_{+-}+T_{--}\right) .
$$

The basic properties of a relation matrix are given by the following result:

Theorem 2.16. Let $T$ be a linear relation in a Krein space $\mathfrak{H}$ such that $\mathfrak{D}(T) \supset \mathfrak{H}_{-}$for a fundamental decomposition (1.1) of $\mathfrak{H}$. Define

$$
\begin{aligned}
& T_{+_{+}}:=P_{+}\left(T \cap\left(\mathfrak{H}_{+} \oplus \mathfrak{H}\right)\right)=\left\{\left(f, P_{+} g\right) \in \mathfrak{H}_{+} \oplus \mathfrak{H}_{+} \mid(f, g) \in T\right\}, \\
& T_{-_{+}}:=P_{+}\left(T \cap\left(\mathfrak{H}_{-} \oplus \mathfrak{H}\right)\right)=\left\{\left(f, P_{+} g\right) \in \mathfrak{H}_{-} \oplus \mathfrak{H}_{+} \mid(f, g) \in T\right\}, \\
& T_{+-}:=P_{-}\left(T \cap\left(\mathfrak{H}_{+} \oplus \mathfrak{H}\right)\right)=\left\{\left(f, P_{-} g\right) \in \mathfrak{H}_{+} \oplus \mathfrak{H}_{-} \mid(f, g) \in T\right\}, \\
& T_{--}:=P_{-}\left(T \cap\left(\mathfrak{H}_{-} \oplus \mathfrak{H}\right)\right)=\left\{\left(f, P_{-} g\right) \in \mathfrak{H}_{-} \oplus \mathfrak{H}_{-} \mid(f, g) \in T\right\},
\end{aligned}
$$

where $P_{ \pm}$are the fundamental projectors belonging to (1.1). Then $T$ is represented by the relation matrix (2.6), whose components are given by (2.8). Furthermore,

(i) $\mathfrak{D}\left(T_{++}\right)=\mathfrak{D}\left(T_{+-}\right)=\mathfrak{D}(T) \cap \mathfrak{H}_{+}, \mathfrak{D}\left(T_{-+}\right)=\mathfrak{D}\left(T_{--}\right)=\mathfrak{H}_{-}$;

(ii) $P_{+} T(0)=T_{++}(0)=T_{-+}(0), P_{-} T(0)=T_{+-}(0)=T_{--}(0)$;

(iii) for every $(f, g) \in T$ there exist $f_{ \pm} \in \mathfrak{H}_{ \pm}, g_{ \pm} \in \mathfrak{H}$ and $l \in T(0)$ such that $f=f_{+}+f_{-}, g=g_{+}+g_{-}+l$ and

$$
\begin{array}{ll}
\left(f_{+}, P_{+} g_{+}\right) \in T_{++}, & \left(f_{-}, P_{+} g_{-}\right) \in T_{-+}, \\
\left(f_{+}, P_{-} g_{+}\right) \in T_{+-}, & \left(f_{-}, P_{-} g_{-}\right) \in T_{--} ;
\end{array}
$$

(iv) $T=\left(T_{++}+T_{+-}\right)+\left(T_{-+}+T_{--}\right)$.

If in addition $T(0)$ is closed and $T(0) \subset \mathfrak{H}_{+}$, then

(v) $\left(T_{++}\right)_{s}=\left(T_{s}\right)_{++}$and $T_{++}(0)=T(0)$;

(vi) $\left(T_{-+}\right)_{s}=\left(T_{s}\right)_{-+}$and $T_{-+}(0)=T(0)$;

(vii) $T_{+-}$is an operator and $T_{+-}=\left(T_{s}\right)_{+-}$;

(viii) $T_{--}$is an operator and $T_{--}=\left(T_{s}\right)_{--}$.

Proof. (i) and (ii) are obvious from the definitions. (iii) follows easily if one defines $f_{ \pm}:=P_{ \pm} f(\in \mathfrak{D}(T))$, chooses $g_{ \pm} \in \mathfrak{H}$ such that $\left(f_{+}, g_{+}\right),\left(f_{-}, g_{-}\right) \in T$, and puts $l:=g-g_{+}-g_{-}$.

The verifications of (2.7) and (iv) are direct computations, which use (i)-(iii) and which can be split into the following parts: $T=P_{+} T+P_{-} T, P_{+} T=T_{++}+T_{-+}$ and similarly for $P_{-} T, T \cap\left(\mathfrak{H}_{+} \oplus \mathfrak{H}\right)=T_{++}+T_{+-}$and similarly for $T \cap\left(\mathfrak{H}_{-} \oplus \mathfrak{H}\right)$, and finally $T=T \cap\left(\mathfrak{H}_{+} \oplus \mathfrak{S}\right)+T \cap\left(\mathfrak{H}_{-} \oplus \mathfrak{H}\right)$. 
If in addition $T(0)$ is closed and in $\mathfrak{H}_{+}$, then $T$ is ortho-complemented and $T_{s}=T \cap\left(T_{\infty}\right)^{\perp}$ an operator (see Proposition 2.3 and Theorem 2.4). From (i) it follows that $T_{+_{+}}(0)=T_{-+}(0)=T(0)$ as well as that $T_{+_{-}}$and $T_{--}$are operators. From the identities

$$
\begin{aligned}
\left(T_{s}\right)_{++} & =P_{+}\left(T_{s} \cap\left(\mathfrak{H}_{+} \oplus \mathfrak{S}\right)\right)=P_{+}\left(T \cap\left(\mathfrak{H}_{+} \oplus T(0)^{\perp}\right)\right), \\
\left(T_{++}\right)_{s} & =T_{++} \cap\left[\left(\left(T_{++}\right)_{\infty}\right)^{\perp} \cap \mathfrak{H}_{+}\right] \\
& =\left[P_{+}\left(T \cap\left(\mathfrak{H}_{+} \oplus \mathfrak{H}\right)\right)\right] \cap\left[\mathfrak{H}_{+} \oplus\left(T(0)^{\perp} \cap \mathfrak{S}_{+}\right)\right]
\end{aligned}
$$

we see that $\left(T_{s}\right)_{++} \subset\left(T_{++}\right)_{s}$. Furthermore, they are both operators with the same domain $\mathfrak{D}(T) \cap \mathfrak{H}_{+}$; thus $\left(T_{s}\right)_{++}=\left(T_{++}\right)_{s}$. (vi)-(viii) are proved similarly.

\section{Dissipative and maximal dissipative relations}

3.1. Dissipative relations. Almost all the properties of a dissipative linear relation $T$ in a Hilbert space are based on the inequality

$$
\|g-z f\| \geqq(\operatorname{Im} \bar{z})\|f\|
$$

for all $(f, g) \in T$, i.e. $C_{-} \subset r(T)$; see [11] (note that our definition of dissipativeness differs from that in [11]). In a general inner product space this inequality is not necessarily true even in the case of operators. In order to get an analogous but weaker form of this result we suppose that our space is a Pontrjagin space.

Theorem 3.1. Let $T$ be a dissipative linear relation in a Pontrjagin space $\mathfrak{H}$ such that $T(0)$ is closed and $\mathfrak{D}(T) \supset \mathfrak{H}_{-}$for a fundamental decomposition (1.1) of $\mathfrak{H}$. Then there exists a constant $c_{T} \geqq 0$ such that

$$
\|g-z f\| \geqq\left(\operatorname{Im} \bar{z}-c_{T}\right)\|f\|
$$

for all $(f, g) \in T$, i.e. $z \in r(T)$ for all $z \in C_{-}$with $\operatorname{Im} \bar{z}>c_{T}$.

Proof. According to Theorem 2.11 we can write $T=S+A$ with $S:=T J$ and $A:=-2 S_{s} P_{-}$. As $\mathfrak{R}\left(P_{-}\right)=\mathfrak{H}_{-}$is finite-dimensional the operator $A$ is bounded; we put $c_{T}:=\|A\|$. (Only here we need the assumption that $\mathfrak{H}$ is a Pontrjagin space.) The operator $A+i c_{T} I$ is $J$-dissipative; indeed, for all $f \in \mathfrak{D}(A)$ we have

$$
\begin{gathered}
\operatorname{Im}\left(A f+i c_{T} f \mid f\right)=\operatorname{Im}(A f \mid f)+c_{T}\|f\|^{2} \\
\geqq-\|A f\|\|f\|+\|A\|\|f\|^{2} \geqq 0 .
\end{gathered}
$$

As $S$ is $J$-dissipative it follows that $S-(\operatorname{Re} z) I$ is also $J$-dissipative. In this case the relation

$$
T-\left(\operatorname{Re} z-i c_{T}\right) I=(S-(\operatorname{Re} z) I)+\left(A+i c_{T} I\right)
$$


is also $J$-dissipative as a sum of two $J$-dissipative relations. So we can use the inequality (3.1) to get

$$
\begin{gathered}
\|g-z f\|\|f\| \geqq \operatorname{Im}(g-z f \mid f) \\
=\operatorname{Im}\left(g-\left(\operatorname{Re} z-i c_{T}\right) f \mid f\right)+\operatorname{Im}\left(\operatorname{Re} z-i c_{T}-z\right)\|f\|^{2} \\
\geqq\left(\operatorname{Im} \bar{z}-c_{T}\right)\|f\|^{2}
\end{gathered}
$$

for all $(f, g) \in T$; hence the result.

The next result gives a sufficient condition for a dissipative linear relation to have dissipative extensions with a given eigenvalue. Later we shall show the converse for symmetric linear relations.

Theorem 3.2. Let $T$ be a dissipative linear relation in an inner product space $\mathfrak{H}$ and let $z \in \boldsymbol{C}$.

(i) If $\mathfrak{R}(T-\bar{z} I)^{\perp}$ contains a non-zero vector $f$ such that $(\operatorname{Im} z)[f \mid f] \geqq 0$, then $T$ has a dissipative extension $T^{\prime}$ in the original space with $z \in \sigma_{p}\left(T^{\prime}\right)$;

(ii) if $\mathfrak{R}(T-\bar{z} I)^{\perp}$ contains a non-zero vector $f$ such that $(\operatorname{Im} z)[f \mid f]>0$, then $T$ has a dissipative extension $T^{\prime}$ in a larger space $\mathfrak{G}^{\prime}$ with $z \in \sigma_{p}\left(T^{\prime}\right)$ and at least one eigenvector does not belong to $\mathfrak{h}$.

Proof. We can follow the proof given in the operator case; see [5]. In case (i) we define $T^{\prime}:=\langle T,(f, z f)\rangle$, where the symbol $\langle.$.$\rangle denotes the subspace spanned$ by the set $\{.$.$\} . Then T^{\prime} \supset T, z \in \sigma_{p}\left(T^{\prime}\right)$ and a direct calculation suffices to show that $T^{\prime}$ is dissipative. In case (ii) we take an element $e$ which does not belong to $\mathfrak{H}$ and define $\mathfrak{H}^{\prime}:=\langle\mathfrak{H}, e\rangle$ with the inner product $[\cdot \mid \cdot]^{\prime}$ :

Then the relation

$$
[g \mid e]^{\prime}:=\left\{\begin{array}{lll}
0 & \text { for } & g \in \mathfrak{H}, \\
-[f \mid f] & \text { for } & g=e .
\end{array}\right.
$$

meets our requirements.

$$
T^{\prime}:=\langle T,(f+e, z(f+e))\rangle
$$

3.2. Maximal dissipative relations. The most interesting extension of a dissipative linear relation in the original space is the maximal one. The following results give some information about maximal dissipative linear relations; for the Hilbert space case see [11].

Theorem 3.3. Let $T$ be a maximal dissipative linear relation in a Krein space G. Then

(i) $T$ is closed;

(ii) $\mathfrak{R}(T-\bar{z} I)^{\perp}$ is positive if $\mathfrak{R}(T-\bar{z} I)$ is non-degenerate for $z \in \boldsymbol{C}_{-}$.

Proof. (i) It is quite clear that the closure of $T$ is a closed dissipative extension of $T$; hence $\bar{T}=T$. (ii) If there exists a non-positive vector $f \in \mathfrak{R}(T-\bar{z} I)^{\perp}$, then $V:=\left\langle C_{z}(T),(0, f)\right\rangle$ is a contractive extension of $C_{z}(T)$. So $F_{z}(V)$ is a dissipative extension of $T$, which implies that $F_{z}(V)=T$ or $V=C_{z}(T)$. Hence $f \in \mathfrak{R}\left(C_{z}(T)\right)=$ $\mathfrak{R}(T-\bar{z} I)$, but $\mathfrak{R}(T-\bar{z} I)$ is non-degenerate and so $f=0$. 
Theorem 3.4. Let $T$ be a dissipative linear relation in a Pontrjagin space $\mathfrak{H}$ such that $T(0)$ is closed and $\mathfrak{D}(T) \supset \mathfrak{H}_{-}$.

(i) If $T$ is maximal, then $\mathfrak{R}(T-z I)=\mathfrak{H}$ for all $z \in \boldsymbol{C}$ with $\operatorname{Im} \bar{z}>c_{T}$;

(ii) if $\mathfrak{R}(T-z I)=\mathfrak{H}$ for $z \in \boldsymbol{C}$ with $\operatorname{Im} \bar{z}>2 c_{T}$, then $T$ is maximal.

Proof. (i) According to the theorems 2.10 and 2.11 we can write $T=S+A$, where $S$ is a maximal $J$-dissipative linear relation and $A$ is a bounded operator with $\|A\|=c_{T}$. Especially $(S-z I)^{-1}$ is an everywhere defined bounded operator with the norm $\leqq 1 / \operatorname{Im} \bar{z}$ for $z \in C_{-}$; see (3.1) and Theorem 2.2. If $\operatorname{Im} \bar{z}>c_{T}$, then

$$
\|A\|=c_{T}<\operatorname{Im} \bar{z} \leqq 1 /\left\|(S-z I)^{-1}\right\|,
$$

and therefore the operator $I+A(S-z I)^{-1}$ has an everywhere defined bounded inverse. In this case

or

$$
T-z I=(S-z I)+A=\left(I+A(S-z I)^{-1}\right)(S-z I)
$$

$$
(T-z I)^{-1}=(S-z I)^{-1}\left(I+A(S-z I)^{-1}\right)^{-1},
$$

but the operator on the right side is everywhere defined and so $\mathfrak{R}(T-z I)=\mathfrak{H}$.

(ii) As in (i) $T=S+A$, where $S$ is $J$-dissipative. Furthermore, by the assumption and by the theorems 2.2 and 3.1 the operator $(T-z I)^{-1}$ is everywhere defined and $\left\|(T-z I)^{-1}\right\| \leqq 1 /\left(\operatorname{Im} \bar{z}-c_{T}\right)$. But then $\|A\|=c_{T}<\operatorname{Im} \bar{z}-c_{T} \leqq 1 /\left\|(T-z I)^{-1}\right\|$, and hence we can proceed as in (i) to get

$$
(S-z I)^{-1}=(T-z I)^{-1}\left(I-A(T-z I)^{-1}\right)^{-1},
$$

which implies $\mathfrak{R}(S-z I)=\mathfrak{H}$. From [11] it follows now that $S$ is maximal $J$-dissipative and so $T$ is maximal by Theorem 2.11 .

Corollary 3.5. Let $T, \mathfrak{H}$ and $z$ be as in Theorem 3.4, part (i). Then $z \in \varrho(T)$ and $\|R(z)\| \leqq\left(\operatorname{Im} \bar{z}-c_{T}\right)^{-1}$ for the resolvent $R(z):=(T-z I)^{-1}$ of $T$.

\section{Symmetric linear relations}

4.1. Eigenvalues, points of regular type. Since every symmetric linear relation $T$ is dissipative, we know from Theorem 3.1 (under the other assumptions made there) that all $z \in \boldsymbol{C}$ with $\operatorname{Im} \bar{z}>c_{T}$ and (for reasons of symmetry) with $\operatorname{Im} \bar{z}<-c_{T}$ are points of regular type of $T$. However, by using better results of the perturbation theory we can improve this. To do so we first examine the eigenvalues of $T$.

The orem 4.1. Let $T$ be a symmetric linear relation in an inner product space $\mathfrak{H}$. Then

(i) $\mathfrak{N}(T-z I)$ and $\mathfrak{N}(T-w I)$ are orthogonal for $z, w \in \sigma_{p}(T), z \neq \bar{w}$;

(ii) $\mathfrak{N}(T-z I)$ is neutral for $z \in \sigma_{p}(T) \backslash \boldsymbol{R}$.

In addition, let $\mathfrak{S}$ be a $\pi_{x}$-space and let $T_{\infty}$ be ortho-complemented. Then 
(iii) the number of the eigenvalues of $T$ belonging to $C_{+}$(resp., $C_{-}$) is at most $\varkappa$;

(iv) $\sigma(T) \backslash \boldsymbol{R}=\sigma_{p}(T) \backslash \boldsymbol{R}$ is symmetric with respect to $\boldsymbol{R}$ for self-adjoint $T$.

Proof. (i) If $f \in \mathfrak{N}(T-z I)$ and $g \in \mathfrak{N}(T-w I)$, then $(f, z f),(g, w g) \in T$ and so

$$
0=[z f \mid g]-[f \mid w g]=(z-\bar{w})[f \mid g] .
$$

(ii) follows immediately from (i). (iii)-(iv) are direct consequences of the theorems 2.7 and 2.9 and of the fact that the corresponding results are valid for operators; see [5]. Note that $T(0)^{\perp}$ is also a $\pi_{x^{\prime}}$-space with $x^{\prime} \leqq x$.

We can now prove the converse of Theorem 3.2 for symmetric linear relations.

Theorem 4.2. Let $T$ be a symmetric linear relation in an inner product space $\mathfrak{H}$ and let $z \in \boldsymbol{C} \backslash \boldsymbol{R}$.

(i) $\mathfrak{R}(T-\bar{z} I)^{\perp}$ contains a non-zero neutral vector iff $T$ has a symmetric extension $T^{\prime}$ in the original space with $z \in \sigma_{p}\left(T^{\prime}\right)$.

(ii) $\mathfrak{R}(T-\bar{z} I)^{\perp}$ contains a non-zero negative vector iff $T$ has a symmetric extension $T^{\prime}$ in a larger space $\mathfrak{H}^{\prime}$, which includes $\mathfrak{H}$ as an ortho-complemented subspace with $\mathfrak{H}^{\perp}$ positive in $\mathfrak{H}^{\prime}$, such that $z \in \sigma_{p}\left(T^{\prime}\right)$ and at least one eigenvector does not belong to $\mathfrak{H}$.

Proof. The other halves of (i) and (ii) follow as in the proof of Theorem 3.2. To show the converse of (i) we take an eigenvector $h$ belonging to $z \in \sigma_{p}\left(T^{\prime}\right)$. Then $h$ is neutral by Theorem 4.1 and belongs to $\mathfrak{R}(T-\bar{z} I)^{\perp}$. Indeed, since $(h, z h) \in T^{\prime}$ and $T^{\prime}$ is symmetric we have

for all $(f, g) \in T \subset T^{\prime}$.

$$
[g-\bar{z} f \mid h]=[g \mid h]-[f \mid z h]=0
$$

For the converse of (ii) we denote by $P$ the orthogonal projector of $\mathfrak{S}^{\prime}$ onto $\mathfrak{S}$ and let $h \notin \mathfrak{H}$ be an eigenvector belonging to $z \in \sigma_{p}\left(T^{\prime}\right)$. Then

$$
0=[h \mid h]=[P h \mid P h]+[(I-P) h \mid(I-P) h]
$$

and $(I-P) h \in \mathfrak{H}^{\perp}$ is positive; hence $P h$ is negative. Furthermore, it belongs to $\Re(T-\bar{z} I)^{\perp}$, because

for all $(f, g) \in T \subset T^{\prime}$.

$$
[g-\bar{z} f \mid P h]=[g \mid h]-[f \mid z h]=0
$$

We remark that if the space $\mathfrak{H}$ in Theorem 4.2 is a Pontrjagin space, then (ii) in Theorem 4.2 can be given the following form: $\mathfrak{R}(T-\bar{z} I)^{\perp}$ contains a non-zero negative vector iff $T$ has a regular symmetric extension $T^{\prime}$ with $z \in \sigma_{p}\left(T^{\prime}\right)$ and at least one corresponding eigenvector does not belong to $\mathfrak{H}$. Here a regular extension means an extension which is defined in a Pontrjagin space $\mathfrak{H}^{\prime} \supset \mathfrak{H}$ with the same number of negative squares as in the original space $\mathfrak{H}$. This remark follows from the facts that then $\mathfrak{H}^{\perp}$ is positive and $\mathfrak{H}$ is ortho-complemented in $\mathfrak{H}^{\prime}$; see [5].

Corollary 4.3. Let $T$ be a symmetric linear relation in a Pontrjagin space $\mathfrak{H}$ and let $z \in \boldsymbol{C} \backslash \boldsymbol{R}$. The subspace $\mathfrak{R}(T-\bar{z} I)^{\perp}$ is positive iff $T$ does not have any regular symmetric extension $T^{\prime}$ with $z \in \sigma_{p}\left(T^{\prime}\right)$. 
Theorem 4.4. Let $T$ be a closed symmetric linear relation in a Pontrjagin space $\mathfrak{H}$ such that $\mathfrak{D}(T) \supset \mathfrak{H}$ - for a fundamental decomposition (1.1) of $\mathfrak{H}$. Then all non-real points $z \notin \sigma_{p}(T)$ are points of regular type of $T$.

Proof. By Theorem 2.11 we have $T_{s}=S_{s}+A$, where $S_{s}$ is a closed $J$-symmetric operator and $A:=-2 S_{s} P_{-}$. As $\mathfrak{R}\left(P_{-}\right)=\mathfrak{H}_{-}$is finite-dimensional the operator $A$ is $S_{s}$-completely continuous and then by [13], Theorem 9.4, all non-real $z \notin \sigma_{p}\left(T_{s}\right)=$ $\sigma_{p}(T)$ are points of regular type for $S_{s}+A=T_{s}$. Theorem 2.7 then implies the result.

4.2. The relation matrix. The relation matrices introduced in Section 2.5 are useful especially in the study of symmetric linear relations and their extensions. As to the operator case, see [5] and [15].

Theorem 4.5. Let $T$ be a closed symmetric linear relation in a Krein space $\mathfrak{H}$ with $\mathfrak{D}(T) \supset \mathfrak{H}_{-}$and let (2.6) with the components (2.8) be a relation matrix of $T$. Then

(i) $T_{++}$is a closed $J$-symmetric linear relation in $\mathfrak{H}_{+}$with $T_{++}(0)=T(0)$;

(ii) $T_{-}$is a continuous $J$-self-adjoint operator in $\mathfrak{H}_{-}$;

(iii) $T_{-+}$is a closed linear relation from $\mathfrak{H}_{-}$into $\mathfrak{H}_{+}$with $T_{-+}(0)=T(0)$, the operator part $\left(T_{-+}\right)_{s}$ is continuous and the J-adjoint $\left(T_{-+}\right)^{*}$ is an operator;

(iv) $T_{+-}$is a continuous operator which admits a continuous closure $\overline{T_{+_{-}}}$with the domain $\overline{\mathfrak{D}(T)} \cap \mathfrak{G}_{+}$, and in addition $\overline{T_{+_{-}}} \subset-\left(T_{-_{+}}\right)^{*}$ and $\left\|T_{+-}\right\| \leqq\left\|\left(T_{-_{+}}\right)_{s}\right\|$;

(v) $T$ is self-adjoint in $\mathfrak{H}$ iff $T_{++}$is $J$-self-adjoint in $\mathfrak{H}_{+}$; in this case $\overline{T_{+_{-}}}=$ $-\left(T_{-+}\right)^{*}$.

Proof. Because $T(0)$ is closed and

$$
T(0) \subset T^{+}(0)=\mathfrak{D}(T)^{\perp} \subset\left(\mathfrak{H}_{-}\right)^{\perp}=\mathfrak{H}_{+},
$$

we can use Theorem 2.16 .

(i) If $(f, g),(h, k) \in T_{++}$, then they are of the form

$$
\begin{array}{lll}
(f, g)=\left(f, P_{+} l\right) \quad \text { with } \quad f \in \mathfrak{H}_{+}, & (f, l) \in T, \\
(h, k)=\left(h, P_{+} m\right) & \text { with } \quad h \in \mathfrak{H}_{+}, \quad(h, m) \in T .
\end{array}
$$

Furthermore, according to Section 1.3 the $J$-inner product $(\cdot \mid \cdot)$ in $\mathfrak{H}_{+}$is equal to the inner product $[\cdot \mid \cdot]$. So we have

$$
\begin{gathered}
(g \mid h)-(f \mid k)=\left[P_{+} l \mid h\right]-\left[f \mid P_{+} m\right] \\
=[l \mid h]-[f \mid m]=0,
\end{gathered}
$$

since $T$ is symmetric. Thus $T_{++}$is $J$-symmetric in $\mathfrak{H}_{+}$. The closedness of $T_{++}$follows in the same way as the closedness of $T_{-+}$; see (iii).

(ii) In a similar manner as in (i) it can be seen that $T_{--}$is $J$-symmetric. Furthermore, $\left(T_{--}\right)^{*}(0)=\mathfrak{D}\left(T_{--}\right)^{(\perp)}=\{0\}$ and so $\left(T_{--}\right)^{*}$ is an operator. But then $T_{--}$ must be $J$-self-adjoint and continuous. 
(iii) As $T_{-+}(0)=T(0)$, the subspace $\left(T_{-+}\right)_{\infty}$ is ortho-complemented by Proposition 2.3 and so we have the decomposition $T_{-+}=\left(T_{-+}\right)_{s}[\dot{+}]\left(T_{-+}\right)_{\infty}$. In addition, $\left(T_{-+}\right)^{*}(0)=\mathfrak{D}\left(T_{-+}\right)^{(\perp)}=\{0\}$, i.e. $\left(T_{-+}\right)^{*}$ is an operator. Let us prove that $T_{-+}$is closed. We can write

$$
T_{-+}=\operatorname{T\cap }\left(\mathfrak{H}_{-} \oplus \mathfrak{H}\right)-T_{--},
$$

because $T \cap\left(\mathfrak{H}_{-} \oplus \mathfrak{H}\right)=T_{-+}+T_{--}$and $T_{--}$is an everywhere defined operator. Let $\left(f_{n}, g_{n}\right)$ be a sequence in $T_{-+}$converging to $(f, g) \in \mathfrak{H}_{-} \oplus \mathfrak{H}_{+}$. Then $\left(f_{n}, g_{n}\right)=$ $\left(f_{n}, k_{n}-T_{--} f_{n}\right)$ with $\left(f_{n}, k_{n}\right) \in T$. The convergence of the sequencies $\left(f_{n}\right)$ and $\left(g_{n}\right)$ and the continuity of $T_{--}$imply that $k_{n}=g_{n}+T_{--} f_{n}$ converges to some $k \in \mathfrak{H}$. Then $\left(f_{n}, k_{n}\right) \rightarrow(f, k) \in T \cap\left(\mathfrak{H}_{-} \oplus \mathfrak{H}\right)$ and especially $f \in \mathfrak{D}\left(T_{--}\right)$. So we get

$$
\left(f_{n}, g_{n}\right)=\left(f_{n}, k_{n}-T_{--} f_{n}\right) \rightarrow\left(f, k-T_{--} f\right),
$$

i.e. $(f, g)=\left(f, k-T_{--} f\right) \in T_{-+}$. Thus $T_{-+}$is closed and by Theorem $2.4\left(T_{-+}\right)_{s}$ is also closed. Being defined everywhere in $\mathfrak{H}_{-}$the operator $\left(T_{-+}\right)_{s}$ is continuous.

(iv) If $\left(f, P_{-} g\right) \in T_{+-}$and $\left(h, P_{+} k\right) \in T_{-+}$, then

$$
\left(P_{-} g \mid h\right)-\left(f \mid-P_{+} k\right)=-[g \mid h]+[f \mid k]=0,
$$

because $T$ is symmetric. Hence $T_{+_{-}} \subset-\left(T_{-+}\right)^{*}$ and so by (iii) $T_{+-}$is an operator. To prove the continuity of $T_{+-}$take an arbitrary vector $f \in \mathfrak{D}\left(T_{+-}\right)$. Then $T_{+-} f \in \mathfrak{H}_{-}=\mathfrak{D}\left(T_{-+}\right)$and so $\left(T_{+-} f, g\right) \in T_{-+}$for some $g \in \mathfrak{H}_{+}$. This element has the decomposition

$$
\left(T_{+-} f, g\right)=\left(T_{+-} f,\left(T_{-+}\right)_{s} T_{+-} f+k\right)
$$

with $k \in T(0)$. As $T_{+-} \subset-\left(T_{-+}\right)^{*}$ and $T(0) \perp \mathfrak{D}\left(T_{+_{-}}\right)$, we have

$$
\begin{gathered}
\left\|T_{+_{-}} f\right\|^{2}=\left(T_{+-} f \mid T_{+_{-}} f\right)=|(f \mid g)| \\
=\left|\left(f \mid\left(T_{-+}\right)_{s} T_{+-} f\right)\right| \leqq\left\|\left(T_{-+}\right)_{s}\right\|\left\|T_{+-} f\right\|\|f\|,
\end{gathered}
$$

which implies $\left\|T_{+-}\right\| \leqq\left\|\left(T_{-_{+}}\right)_{s}\right\|$. The properties of the extension $\overline{T_{+-}}$are obvious.

(v) Suppose that $T$ is self-adjoint. We should prove the inclusion $\left(T_{++}\right)^{*} \subset$ $T_{++}$. For this, let $(h, k) \in\left(T_{++}\right)^{*}$ be arbitrary. If we can find a vector $g \in \mathfrak{H}_{-}$ such that $(h, k+g) \in T^{+}=T$, then $(h, k)=\left(h, P_{+}(k+g)\right)$, i.e. $(h, k) \in T_{++}$. Using (i), Proposition 2.3 and (iv) we get

$$
\begin{aligned}
\mathfrak{D}\left(\left(T_{++}\right)^{*}\right) & \subset \mathfrak{D}\left(\left(T_{++}\right)^{*}\right)^{\perp \perp}=\overline{T_{++}}(0)^{\perp}=T(0)^{\perp} \\
& =\overline{\mathfrak{D}(T)} \cap \mathfrak{H}_{+}=\mathfrak{D}\left(\overline{T_{+-}}\right)
\end{aligned}
$$

(here the orthogonal companions are in $\mathfrak{H}_{+}$). By choosing $g:=\overline{T_{+-}} h$ one can, with the help of (i), (iv) and Theorem 2.16, verify that $(h, k+g) \in T^{+}$as we wanted.

Suppose that $T_{++}$is $J$-self-adjoint in $\mathfrak{H}_{+}$. We should prove $T^{+} \subset T$. Take an arbitrary $(h, k) \in T^{+}$. Using the assumption, (iv) and some calculations we see that $\left(h, P_{+} k\right)$ is in $T_{++}+T_{-+}$. Similarly $\left(h, P_{-} k\right)$ is seen to be in $T_{+-}+T_{--}$. By (2.7) the element $(h, k)$ is then in $T$. 
Finally we show that $\overline{T_{+-}}=-\left(T_{-+}\right)^{*}$ in case $T$ is self-adjoint. By (iv) it is enough to prove the inclusion $\mathfrak{D}\left(\left(T_{-+}\right)^{*}\right) \subset T(0)^{\perp} \cap \mathfrak{H}_{+}$. As above we get

$$
\mathfrak{D}\left(\left(T_{-+}\right)^{*}\right) \subset \mathfrak{D}\left(\left(T_{-+}\right)^{*}\right)^{\perp \perp}=\overline{T_{-+}}(0)^{\perp}=T(0)^{\perp} .
$$

The orem 4.6. Let $T$ be a closed symmetric linear relation in a Pontrjagin space $\mathfrak{H}$ with $\mathfrak{D}(T) \supset \mathfrak{H}_{-}$and with the relation matrix (2.6) defined by (2.8). A linear relation $T^{\prime}$ is a regular self-adjoint extension of $T$ iff it is represented by the relation matrix

$$
\left[\begin{array}{cc}
T_{++}^{\prime} & T_{-+}+\{0\} \oplus T_{++}^{\prime}(0) \\
-\left.\left(T_{-+}\right)^{*}\right|_{\mathcal{D}\left(T_{++}^{\prime}\right)} & T_{--}
\end{array}\right],
$$

where $T_{++}^{\prime}$ is a J-self-adjoint extension of $T_{++}$.

Proof. $1^{\circ}$ Suppose that $T^{\prime}$ is a regular self-adjoint extension of $T$. Then $T^{\prime}$ is a self-adjoint relation in a Pontrjagin space $\mathfrak{H}^{\prime} \supset \mathfrak{H}$, which has as many negative squares as $\mathfrak{H}$, and $T^{\prime}$ can be represented by the relation matrix

$$
\left[\begin{array}{ll}
T_{++}^{\prime} & T_{-+}^{\prime} \\
T_{+-}^{\prime} & T_{--}^{\prime}
\end{array}\right]
$$

where the components are defined as in (2.8). Furthermore, by Theorem $4.5 T_{++}^{\prime}$ is $J$-self-adjoint in $\mathfrak{H}_{+}^{\prime} \supset \mathfrak{S}_{+}$and $\overline{T_{+-}^{\prime}}=-\left(T_{-+}^{\prime}\right)^{*}$. Note that $\mathfrak{S}^{\prime}$ has a fundamental decomposition $\mathfrak{S}^{\prime}=\mathfrak{H}_{+}^{\prime}[\dot{+}] \mathfrak{H}_{-}^{\prime}$ with $\mathfrak{H}_{+}^{\prime} \supset \mathfrak{H}_{+}$and $\mathfrak{S}_{-}^{\prime}=\mathfrak{H}_{-}$; the corresponding fundamental projectors are denoted by $P_{ \pm}^{\prime}$.

We show first that $T_{++}^{\prime}$ is an extension of $T_{++}$. For this let $(f, g) \in T_{++}=$ $P_{+}\left(T \cap\left(\mathfrak{H}_{+} \oplus \mathfrak{H}\right)\right)$ be arbitrary. Then $f \in \mathfrak{H}_{+}$and $(f, g)=\left(f, P_{+} k\right)$ for some $k$ such that $(f, k) \in T \subset T^{\prime}$. As $\left.P_{ \pm}^{\prime}\right|_{\mathfrak{S}}=P_{ \pm}$, we get

i.e. $T_{++} \subset T_{++}^{\prime}$.

$$
(f, g)=\left(f, P_{+} k\right) \in P_{+}^{\prime}\left(T^{\prime} \cap\left(\mathfrak{S}_{+}^{\prime} \oplus \mathfrak{S}^{\prime}\right)\right)=T_{++}^{\prime},
$$

As above, it can be seen that $T_{-+} \subset T_{-+}^{\prime}, T_{+-} \subset T_{+-}^{\prime}$ and $T_{--} \subset T_{--}^{\prime}$. Furthermore, $T_{--}$and $T_{--}^{\prime}$ are operators defined throughout in $\mathfrak{H}_{-}=\mathfrak{H}_{-}^{\prime}$; hence $T_{--}=T_{--}^{\prime}$.

Let us prove the identity $T_{-+}^{\prime}=T_{-+}+\{0\} \oplus T_{++}^{\prime}(0)$. From Theorem 2.16 and from above we get the inclusion $\supset$. Furthermore, it is easy to see that the linear relations on the left and right side of the desired identity have the same domain $\mathfrak{H}_{-}$and their multi-valued parts are equal. These facts imply the equation we wanted.

Finally, we must show $T_{+-}^{\prime}=-\left.\left(T_{-+}\right)^{*}\right|_{\mathfrak{D}\left(T_{++}^{\prime}\right)}$, where the adjoint is taken in the extension space. A little calculation gives

$$
\begin{aligned}
& \left(T_{-+}^{\prime}\right)^{*}=\left(T_{-+}\right)^{*} \cap\left(T_{++}^{\prime}(0)^{\perp} \oplus \mathfrak{H}_{-}\right) \\
& \left.=\left(T_{-+}\right)^{*} \cap\left(\overline{\mathfrak{D}\left(T_{++}^{\prime}\right.}\right) \oplus \mathfrak{S}_{-}\right)=\left(T_{-+}\right)^{*} \mid \overline{\mathfrak{D}\left(T_{++}^{\prime}\right)} .
\end{aligned}
$$

On the other hand, $\overline{T_{+-}^{\prime}}=-\left(T_{-+}^{\prime}\right)^{*}$, and putting these together one gets the desired result. 
$2^{\circ}$ Suppose $T_{++}^{\prime}$ is a $J$-self-adjoint extension of $T_{++}$in a Hilbert space $\mathfrak{H}_{+}^{\prime}$ extending $\mathfrak{H}_{+}$. Then the space $\mathfrak{H}^{\prime}:=\mathfrak{H}_{+}^{\prime} \oplus \mathfrak{H}_{-}$equipped with the usual linear and inner product structure is a $\pi_{x}$-space with a fundamental decomposition

$$
\mathfrak{S}^{\prime}:=\mathfrak{S}_{+}^{\prime}[+] \mathfrak{H}_{-} .
$$

We should show that the linear relation $T^{\prime}$ defined via the relation matrix (4.1) is a self-adjoint extension of $T$.

We verify first that $-\left.\left(T_{-+}\right)^{*}\right|_{\mathcal{D}\left(T_{++}^{\prime}\right)}$ is an operator extension of $T_{+-}$. As $\mathfrak{D}\left(T_{-+}\right)=\mathfrak{H}_{-}$we see that $\left(T_{-+}\right)^{*}$ is an operator. Let $f \in \mathfrak{D}\left(T_{+-}\right)$; then $(k \mid f)=$ $-\left(h \mid T_{+-} f\right)$ for all $(h, k) \in T_{-+}$by Theorem 2.16. This means $\left(f,-T_{+-} f\right) \in$ $\left(T_{-+}\right)^{*}$, i.e. $T_{+-} \subset-\left(T_{-+}\right)^{*}$. Furthermore, $\mathfrak{D}\left(T_{+_{-}}\right)=\mathfrak{D}\left(T_{++}\right) \subset \mathfrak{D}\left(T_{++}^{\prime}\right)$, and so we get the desired result.

To prove the inclusion $T \subset T^{\prime}$, let $(f ; g) \in T$ be arbitrary. Then by Theorem $2.16 g=g_{1}+g_{2}$ and

$$
\begin{aligned}
& \left(f, g_{1}\right)=\left(f_{1}, g_{11}\right)+\left(f_{2}, g_{12}\right), \\
& \left(f, g_{2}\right)=\left(f_{1}, g_{21}\right)+\left(f_{2}, g_{22}\right)
\end{aligned}
$$

with $\left(f_{1}, g_{11}\right) \in T_{++} \subset T_{++}^{\prime},\left(f_{2}, g_{12}\right) \in T_{-+},\left(f_{1}, g_{21}\right) \in T_{+-} \subset-\left.\left(T_{-+}\right)^{*}\right|_{\mathcal{D}\left(T_{++}^{\prime}\right)}$ and $\left(f_{2}, g_{22}\right) \in T_{--}$; hence

$$
(f, g)=\left(f, g_{1}+g_{2}\right) \in\left(T_{++}^{\prime}+T_{-+}\right)+\left(-\left.\left(T_{-+}\right)^{*}\right|_{\mathfrak{D}\left(T_{++}^{\prime}\right)}+T_{--}\right) \subset T^{\prime} .
$$

In order to show the symmetry of $T^{\prime}$, we decompose an arbitrary $(f, g) \in T^{\prime}$ analogously to get

$$
\begin{aligned}
{[g \mid f] } & =\left(g_{11} \mid f_{1}\right)+\left(g_{12} \mid f_{1}\right)-\left(g_{21} \mid f_{2}\right)-\left(g_{22} \mid f_{2}\right) \\
& =\left(f_{1} \mid g_{11}\right)-\left(f_{2} \mid g_{21}\right)+\left(f_{1} \mid g_{12}\right)-\left(f_{2} \mid g_{22}\right) \\
& =[f \mid g] ;
\end{aligned}
$$

i.e. $T^{\prime}$ is symmetric. Here we have used the following facts: $T_{++}^{\prime} \subset\left(T_{++}\right)^{+}$, $\left(f_{2}, g_{12}\right) \in T_{-+},\left(f_{1},-g_{21}\right) \in-\left(T_{-+}\right)^{*}$ and $T_{--} \subset\left(T_{--}\right)^{*}$.

Finally, we must show the inclusion $T^{\prime} \subset \subset T^{\prime}$. For this we need the identity $\mathfrak{D}\left(T_{++}^{\prime}\right)=\mathfrak{D}\left(\left.\left(T_{-+}^{\prime}\right)^{*}\right|_{\mathfrak{D}\left(T_{++}^{\prime}\right)}\right)$, for which in turn we need the fact that $\mathfrak{D}\left(\left(T_{-+}^{\prime}\right)^{*}\right)$ is closed. To prove that fact, one can argue as in the proof of Theorem 4.5 to show that $\left\|\left(T_{-+}\right)^{*} f\right\| \leqq\left\|\left(T_{-+}\right)_{s}\right\|\|f\|$, i.e. $\left(T_{-+}\right)^{*}$ is continuous, which is enough. Then

$$
\mathfrak{D}\left(\left(T_{-_{+}}\right)^{*}\right)=\mathfrak{D}\left(\left(T_{-+}\right)^{*}\right)^{\perp \perp}=T_{-_{+}}(0)^{\perp}=T(0)^{\perp} \supset T^{\prime}(0)^{\perp} \supset \mathfrak{D}\left(T_{++}^{\prime}\right),
$$

from which the identity mentioned above follows.

Let $P_{ \pm}^{\prime}$ be the fundamental projectors belonging to the decomposition (4.2) and take an arbitrary $(h, k) \in T^{\prime+}$. Then a little calculation shows that $\left(h, P_{+}^{\prime} k\right) \in$ $T_{++}^{\prime}+T_{-+}$and analogously $\left(h, P_{-}^{\prime}\right) \in-\left.\left(T_{-+}\right)^{*}\right|_{\mathcal{D}\left(T_{++}^{\prime}\right)}+T_{--}$, i.e. $(h, k) \in T^{\prime}$. This completes the proof.

Corollary 4.7. Every closed symmetric linear relation $T$ in a Pontrjagin space $\mathfrak{H}$ with $\mathfrak{D}(T) \supset \mathfrak{S}_{-}$admits regular self-adjoint extensions.

We can put the previous theorem in a perturbational form. 
Theorem 4.8. Let $T$ and $\mathfrak{S}$ be as in Theorem 4.6. Every regular self-adjoint extension $T^{\prime}$ of $T$ is of the form $T^{\prime}=S^{\prime}+A$, where $S^{\prime}$ is a J-self-adjoint linear relation and $A$ is a continuous operator with $\|A\| \leqq c_{T}$.

Proof. From Theorem 4.6 we know that $T^{\prime}$ is represented by the relation matrix (4.1). Define $S^{\prime}:=T_{++}^{\prime}+T_{--}$; as $T_{++}^{\prime}$ and $T_{--}$are $J$-self-adjoint it is easy to see that $S^{\prime}$ has the same property. Let

$$
A:=-\left.\left(T_{-+}\right)^{*}\right|_{\mathcal{D}\left(T_{++}^{\prime}\right)}+\left(T_{-+}\right)_{s} .
$$

Then $A$ is an operator with $\mathfrak{D}(A)=\mathfrak{D}\left(T^{\prime}\right)$.

To prove the continuity of $A$, let $f \in \mathcal{D}(T)$ be arbitrary. Then

$$
A f=-\left(T_{-+}\right)^{*} f_{1}+\left(T_{-+}\right)_{s} f_{2}
$$

with $f_{1} \in \mathfrak{D}\left(T_{++}^{\prime}\right)$ and $f_{2} \in \mathfrak{D}\left(T_{-+}\right)$. This implies

$$
\begin{aligned}
\|A f\|^{2} & =\left\|\left(T_{-+}\right)^{*} f_{1}\right\|^{2}+\left\|\left(T_{-+}\right)_{s} f_{2}\right\|^{2} \\
& \leqq\left\|\left(T_{-+}\right)_{s}\right\|^{2}\left(\left\|f_{1}\right\|^{2}+\left\|f_{2}\right\|^{2}\right) \\
& =\left\|\left(T_{-+}\right)_{s}\right\|^{2}\|f\|^{2}
\end{aligned}
$$

(see the proof of the preceding theorem). So we must prove that $\left\|\left(T_{-+}\right)_{s}\right\| \leqq$ $2\left\|S_{s} P_{-}\right\|=: c_{T}$ (for the definition of $S_{s}$, see Theorem 2.11). With Theorem 2.16 and a little calculation we derive

$$
\left(T_{-+}\right)_{s}=\left(T_{s}\right)_{-+}=\left(S_{s} J\right)_{-+}=\left.P_{+} S_{s} J\right|_{\mathfrak{S}_{-}} \subset-P_{+} S_{s} P_{-},
$$

which implies the desired inequality.

Finally, to verify that $T^{\prime}=S^{\prime}+A$ one can proceed as follows: With the help of the relation matrix of $T^{\prime}$ it is seen that $T^{\prime} \subset S^{\prime}+A$. Furthermore, $\mathcal{D}\left(S^{\prime}\right)=$ $\mathfrak{D}\left(T^{\prime}\right)=\mathfrak{D}(A)$ and $T^{\prime}(0)=\left(S^{\prime}+A\right)(0)$. These facts together imply the result.

Corollary 4.9. Let $T$ and $\mathfrak{H}$ be as in Theorem 4.6 and let $T^{\prime}$ be an arbitrary regular self-adjoint extension of $T$. Then

(i) $\sigma\left(T^{\prime}\right) \subset \boldsymbol{C}_{T}:=\left\{z \in \boldsymbol{C}|| \operatorname{Im} z \mid \leqq c_{T}\right\}$;

(ii) $\left\|\left(T^{\prime}-z I\right)^{-1}\right\| \leqq\left(\operatorname{Im} z-c_{T}\right)^{-1}$ for $z \notin \boldsymbol{C}_{T}$;

(iii) $\mathfrak{R}(T-z I)^{\perp}$ is positive for $z \notin \boldsymbol{C}_{T}$.

For the proof, see Corollary 3.5 and Corollary 4.3.

4.3. The deficiency spaces. Let $T$ be a relation in an inner product space $\mathfrak{H}$. Define $\mathfrak{N}_{z}:=\mathfrak{N}_{z}(T):=\mathfrak{R}(T-\bar{z} I)^{\perp}$ and $M_{z}:=M_{z}(T):=\left\{(h, k) \in T^{+} \mid k=z h\right\}$ for $z \in C$; the latter is called a deficiency space of $T$ in [11]. In studying these spaces we restrict ourselves to Pontrjagin spaces.

Theorem 4.10. Let $T$ be a closed symmetric linear relation in a Pontrjagin space $\mathfrak{H}$ with $\mathfrak{D}(T) \supset \mathfrak{H}_{-}$for a fundamental decomposition (1.1) of $\mathfrak{H}$. Then 
(i) $M_{z}$ is a continuous operator with $\mathfrak{D}\left(M_{z}\right)=\mathfrak{R}\left(M_{z}\right)=\mathfrak{N}_{z}$ for all $z \in \boldsymbol{C} \backslash \boldsymbol{R}$;

(ii) $M_{z}$ and $\mathfrak{N}_{z}$ are Hilbert spaces with respect to $[\cdot \mid \cdot]$ for all $z \notin C_{T}$;

(iii) $\operatorname{dim} M_{z}=\operatorname{dim} \mathfrak{N}_{z}$ is constant for all $z \in C_{+} \backslash \sigma_{p}(T)$ and similarly for all $z \in C_{-} \backslash \sigma_{p}(T)$;

(iv) $T^{+}=T+M_{z}+M_{\bar{z}}$ for all $z \notin C_{T}$.

Proof. (i) is obvious and (ii) follows from Corollary 4.9. Clearly $\operatorname{dim} M_{z}=$ $\operatorname{dim} \mathfrak{N}_{z}=\operatorname{dim} \mathfrak{R}(T-\bar{z} I)^{(\perp)}$, and the constancy of this dimension follows from Theorem 4.4 as in the case of Hilbert space operators; see [1], Nr. 100. (iv) is a direct calculation.

The cardinal number $\mathfrak{n}_{+}:=\mathfrak{n}_{+}(T):=\operatorname{dim} M_{\bar{z}}(T)$ for $z \in C_{+} \backslash \sigma_{p}(T)$ is called the upper defect number of $T$; similarly $\mathrm{n}_{-}:=\mathfrak{n}_{-}(T):=\operatorname{dim} M_{\bar{z}}(T)$ for $z \in C_{-} \backslash \sigma_{p}(T)$ is called the lower defect number of $T$. The previous results show that $T$ is maximal symmetric/self-adjoint iff $n_{+}=0$ or $n_{-}=0 / n_{+}=n_{-}=0$.

A mapping $U: \mathfrak{H} \rightarrow \mathfrak{H}$ is called a conjugation if $U^{2}=I$ and $[U f \mid U g]=[g \mid f]$ for all $f, g \in \mathfrak{H}$. A linear relation $T$ in $\mathfrak{H}$ is said to be real with respect to the conjugation $U$, if $(f, g) \in T$ implies $(U f, U g) \in T$. A useful criteria for the equality $n_{+}=n_{-}$is given by the following result:

Theorem 4.11. Let $T$ and $\mathfrak{G}$ be as in Theorem 4.10. If $T$ is real with respect to a conjugation, then the defect numbers of $T$ are equal.

As the spaces $\mathfrak{N}_{ \pm i a}$ with $a>c_{T}$ are Hilbert spaces, we can use the proof given for the Hilbert space operators; see e.g. [12], Theorem XII.4.18.

As in the Hilbert space case, the sums in (iv) of Theorem 4.10 are orthogonal for $z \in\{i,-i\}$, but it can very well happen here that $\pm i \in C_{T}$. In order to get orthogonal sums also in this case, we need to modify the inner product:

Theorem 4.12. Let $T$ and $\mathfrak{H}$ be as in Theorem 4.10 and let $a>c_{T}$. Define

$$
[(f, g) \mid(h, k)]_{+}:=a^{2}[f \mid h]+[g \mid k]
$$

for all $(f, g),(h, k) \in T^{+}$. Then $T^{+}$is a $\pi_{\varkappa}$-space with the inner product $[\cdot \mid \cdot]_{+}$and

$$
T^{+}=T[\dot{+}]_{+} M_{i a}[\dot{+}]_{+} M_{-i a} \text {. }
$$

The proof follows the same lines as in the operator case; see [20].

\section{References}

[1] AChieser, N. I., and I. M. Glasmann: Theorie der linearen Operatoren im Hilbert-Raum. Mathematische Lehrbücher und Monographien I. IV, Akademie-Verlag, Berlin, 1968.

[2] Arens, R.: Operational calculus of linear relations. - Pacific J. Math. 11, 1961, 9-23.

[3] Atkinson, F. W., W. N. Everitt, and K. S. Ong: On the $m$-coefficient of Weyl for a differential equation with an indefinite weight function. - Proc. London Math. Soc. (3) 29, 1974, 368-384. 
[4] Bennewitz, C.: Symmetric relations on a Hilbert space. - Conference on the Theory of Ordinary and Partial Differential Equations, Dundee, Scotland, 1972. Lecture Notes in Mathematics 280, Springer-Verlag, Berlin-Heidelberg-New York, 1972, 212-218.

[5] BognÁR, J.: Indefinite inner product spaces. - Ergebnisse der Mathematik und ihrer Grenzgebiete 78, Springer-Verlag, Berlin-Heidelberg-New York, 1974.

[6] Coddington, E. A.: Extension theory of formally normal and symmetric subspaces. - Mem. Amer. Math. Soc. 134, 1973, 1-80.

[7] Coddington, E. A.: Self-adjoint subspace extensions of nondensely defined symmetric operators. - Advances in Math. 14, 1974, 309-332.

[8] Codpington, E. A.: Spectral theory of ordinary differential operators. - Spectral Theory and Differential Equations, Proceedings of the Symposium held at Dundee, Scotland, 1974. Lecture Notes in Mathematics 448, Springer-Verlag, Berlin-Heidelberg- New York, 1975, 1-24.

[9] Coddington, E. A.: Self-adjoint problems for nondensely defined ordinary differential operators and their eigenfunction expansions. - Advances in Math. 15, 1975, 1-40.

[10] DAHo, K., and H. LANGER: Sturm-Liouville operators with an indefinite weight function. To appear.

[11] Disksma, A., and H. S. V. DE SNoo: Self-adjoint extensions of symmetric subspaces. - Pacific J. Math. 54, 1974, 71-100.

[12] Dunford, N., and J. T. Schwartz (with the assistance of W. G. Bade and R. G. Bartle): Linear operators, Part II: Spectral theory, Self adjoint operators in Hilbert space. Pure and applied mathematics VII, Interscience Publishers (John Wiley \& Sons), New York-London-Sydney, 1963.

[13] Gohberg, I. C., and M. G. KreĬN: The basic propositions on defect numbers, root numbers and indices of linear operators. - Amer. Math. Soc. Transl. (2) 13, 1960, 185-264.

[14] Kato, T.: Perturbation theory for linear operators. - Die Grundlehren der mathematischen Wissenschaften 132, Springer-Verlag, Berlin-Heidelberg-New York, 1966.

[15] Krein, M. G., and G. K. LANGER: Defect subspaces and generalized resolvents of an Hermitian operator in the space $\Pi_{\varkappa}$. - Functional Anal. Appl. 5, 1971, 136-146.

[16] Krein, M. G., and JU. L. ŠMUL'JAN: I-polar representation of plus-operators. - Amer. Math. Soc. Transl. (2) 85, 1969, 115-143.

[17] LANGER, H.: Zur Spektraltheorie verallgemeinerter gewöhnlicher Differentialoperatoren zweiter Ordnung mit einer nichtmonotonen Gewichtsfunktion. - Ber. Univ. Jyväskylä Math. Inst. 14, 1972, 1-58.

[18] LANGer, H., and B. TeXtorius: On generalized resolvents and $Q$-functions of symmetric linear relations (subspaces) in Hilbert space. - Pacific J. Math. (to appear).

[19] Pleijel, Å.: A survey of spectral theory for pairs of ordinary differential operators. - Spectral Theory and Differential Equations, Proceedings of the Symposium held at Dundee, Scotland, 1974. Lecture Notes in Mathematics 448, Springer-Verlag, Berlin-Heidelberg-New York, 1975, 256-272.

[20] SoRJonen, P.: Über gewisse Tripel von Pontrjaginräumen. - Math. Nachr. 63, 1974, 213-221.

\author{
University of Jyväskylä \\ Department of Mathematics \\ SF-40 100 Jyväskylä 10 \\ Finland
}

Received 30 March 1978 